\title{
Actions entreprises pour la réduction des doses reçues par les travailleurs des centrales PWR*
}

\author{
P. JEANSON $\star \star$ \\ (Manuscrit reçu le 1er décembre 1982)
}

\begin{abstract}
RÉSUME
La réduction des doses subies par les personnes travaillant dans les centrales nucléaires est un objectif permanent d'Electricité de France. De nombreuses actions sont entreprises simultanement ; certaines d'entre-elles sont menées en collaboration avec le CEA. Les études théoriques portent essentiellement sur la formation et le transfert des produits de corrosion et de fission dans le circuit primaire des réacteurs à eau sous pression ainsi que sur les moyens propres à réduire l'activité déposée. Les études pratiques portent sur la conception des materiels ou des locaux et sur leurs règles d'installation ainsi que sur le conditionnement chimique du fluide primaire. Elles portent également sur les conditions d'entretien de tous les composants; pour les plus importants, des études d'outillage sont entreprises. Enfin, la réalisation d'une dosimétrie journalière permet de mieux connaître la répartition des doses collectives et, par suite, de diriger judicieusement les actions à entreprendre.
\end{abstract}

\section{ABSTRACT}

The reduction of doses to nuclear power plant workers is a permanent aim of Electricité de France. Many actions are undertaken simultaneously, some of them jointly with the Commissariat à l'energie atomique. The theoretical studies bear mainly on the formation and transfer of corrosion and fission products in the primary circuit of PWRs and on the procedures to lower the deposited activity. The practical studies bear on the design of materials and buildings and the rules of installation as well as on the chemical conditioning of the primary fluid. They also bear on the conditions of maintenance of all the components ; for the main ones, special tools are designed. Finally, operating a daily dosimetry makes it possible to know the distribution of collective doses better and therefore to judiciously direct the actions to be undertaken.

\section{INTRODUCTION}

La réduction des doses reçues par le personnel des centrales nucléaires est un souci permanent d'Electricité de France. L'effort entrepris dans ce

* PWR (Pressurized water reactor) : réacteur à eau sous pression.

** Electricité de France. Service de la production technique, Département Sécurité, radioprotection, environnement, BP 114, 93203 Saint-Denis Cedex 1. 
domaine est très vaste du fait des nombreuses directions dans lesquelles il s'exerce et de l'importance des moyens mis en œuvre. II est justifié par l'ampleur du programme nucléaire français et par le souci de maintenir les doses des travailleurs aussi bas qu'il est raisonnablement possible. II est accompli le plus souvent en collaboration avec le CEA, Framatome et Westinghouse. Cet article tente de faire une synthèse des principales actions menées dans ce domaine sans, toutefois, traiter le cas des situations accidentelles. II fait suite et complète l'article paru en 1978 sur le même sujet [2].

La réduction des doses reçues par le personnel nécessite une connaissance approfondie des mécanismes de formation des sources actives qui en sont responsables afin de mettre en évidence les paramètres sur lesquels il est possible d'agir pour en réduire l'activité. D'autre part, pour une activité donnée des sources, la réduction des doses s'opère par une réduction du temps d'exposition aux rayonnements ou par une réduction du débit de dose (usage d'écrans absorbants ou travail à distance) et, dans une certaine mesure, par la décontamination préalable des circuits actifs.

\section{ETUDE DES SOURCES ACTIVES DANS LES REACTEURS PWR}

Les sources peuvent être classées, selon leur origine ou leur mode de formation, en deux catégories :

- les produits de fission formés dans le combustible qui, lui-même émet des neutrons ;

- les produits d'activation formés par le flux neutronique issu du combustible.

Les produits de fission (dans le cas de ruptures de gaines) et certains produits d'activation sont véhiculés par le fluide primaire (eau sous pression). Une partie d'entre eux se dépose sur la paroi interne des équipements et occasionne ainsi, sur l'ensemble des circuits actifs, des dépôts dont l'activité devient rapidement supérieure à celle de l'eau. D'autre part, les fuites inévitables des circuits peuvent entraîner une contamination des sols et de l'air des locaux. Cette observation de la nature physique des sources ou de leur emplacement géographique permet de distinguer plusieurs origines aux doses subies par le personnel: combustible, air des locaux, structures métalliques activées, fluides, dépôts sur la paroi interne des tuyauteries, etc.

Lorsque l'on estime l'importance relative de chacune d'elles, il apparaît que $90 \%$ des doses subies par le personnel sont dues aux dépôts formés sur les parois internes des équipements.

Le principal responsable est le cobalt 60 qui en induit à lui seul environ $60 \%$ (en l'absence de ruptures de gaines). L'expérience acquise en matière de ruptures de gaines ${ }^{(1)}$ pour un fonctionnement aux limites régle-

(1) La rupture de la gaine métallique qui entoure les pastilles de combustible laisse échapper dans l'eau primaire certains produits de fission, ceux-ci étant normalement contenus à l'intérieur de la gaine. 
mentaires montre que la présence de produits de fission n'a qu'une importance marginale sur les doses du personnel.

Ceci montre l'intérêt de l'étude des sources afin de mettre en évidence les points sur lesquels l'effort doit porter en priorité et explique d'autre part pourquoi l'étude de la réduction des dépôts actifs occupe une place prioritaire.

\subsection{Dépôts de produits de corrosion}

\subsubsection{Etudes théoriques - Code PACTOLE}

La formation et le transfert des produits de corrosion activés dans le circuit primaire des réacteurs PWR constitue la base de ce problème. Cette étude, menée depuis de nombreuses années au Commissariat à l'énergie atomique a abouti à la mise au point d'un code de calcul (PACTOLE) permettant de prévoir l'évolution dans le temps de l'activité surfacique des dépôts en fonction des principaux paramètres régissant ces phénomènes :

- nature et teneur en cobalt des matériaux ;

- puissance du réacteur ;

- vitesse d'écoulement du fluide ;

- température, $\mathrm{pH}$, teneur en hydrogène de l'eau primaire.

Bien que d'autres paramètres interviennent, la comparaison périodique entre les résultats de calcul et les mesures sur des réacteurs en service, l'analyse systématique des résultats, la recherche permanente de la compréhension des phénomènes physiques permettent d'affiner ce code dont les résultats sont déjà satisfaisants. Les gains escomptés sur la dose collective, cités dans la suite du texte, sont des gains théoriques calculés au moyen de ce code.

\subsubsection{Etudes en laboratoire}

Parmi les paramètres pris en compte figure la solubilité des produits de corrosion, dont la connaissance encore imparfaite a justifié la réalisation d'un programme d'études. Ce programme se poursuit, essentiellement pour rechercher l'évolution de la solubilité en fonction de la température et l'existence éventuelle d'un minimum dans la gamme des températures rencontrées en réacteur. Ce dernier point revêt une importance particulière pour la compréhension des phénomènes physiques.

\subsubsection{Etudes sur boucles d'essais}

Etant donné la complexité des problèmes de formation des dépôts, certaines études expérimentales ont dû être réalisées dans des boucles reproduisant partiellement les conditions réelles de fonctionnement.

Pour EdF : - la boucle BECO permet d'effectuer des études du relâchement et de redéposition pour les matériaux utilisés dans les circuits des réacteurs (inconel, acier austénitique, stellite) en fonction du temps et des caractéristiques chimiques de l'eau primaire ;

- la boucle BOUCOR permet de mesurer le taux de corrosion de l'inconel 600 et 690 pour différents états de surface et traitements thermiques. 
Par ailleurs, le CEA étudie dans les boucles BIHAN et HPZ les transferts de masse en fonction de la chimie et se propose d'étudier certains aspects du relâchement des matériaux dans la boucle CORELE. Les boucles BIHAN, IRENE et CIRENE sont aussi utilisées pour observer les dépôts sur le combustible et la corrosion du gainage en présence d'ébullition nuclée.

\subsubsection{Mesures sur réacteurs}

Parallèlement à ces études en boucles, des mesures spectrométriques sur les réacteurs en service, portant sur l'activité de l'eau et les dépôts du circuit primaire, donnent une connaissance détaillée de l'état de certains réacteurs, ce qui conduit occasionnellement à mettre en évidence une anomalie et ses causes. Ces mesures sont nécessaires, par les données expérimentales qu'elles apportent, pour approfondir la réflexion et les connaissances sur le comportement des produits de corrosion activés.

De plus, tous les réacteurs PWR font l'objet d'un suivi systématique moins approfondi mais plus vaste de l'activité des principaux circuits (mesures de débit de dose) qui est utilisé pour en suivre l'évolution et, éventuellement, déclencher des investigations plus détaillées.

En complément, les niveaux d'activité de tous les circuits actifs de Tihange et prochainement de Dampierre sont suivis en détail (1000 points de mesure environ). Ces résultats constituent un élément de base essentiel pour les études prévisionnelles de doses collectives. D'autre part, ils sont précieux pour mieux tenir compte, dès la conception des nouvelles tranches PWR, des problèmes de protection du personnel.

\subsubsection{Etudes particulières}

Des efforts sont actuellement poursuivis sur des points particuliers :

a) Suivi de charge

Compte tenu de la part progressivement prépondérante des centrales nucléaires dans la production nationale, les réacteurs PWR sont dès à présent amenés à faire varier, de façon programmée, leur puissance en fonction de la prévision de la consommation. C'est ce que l'on appelle le suivi de charge. Ceci semble entraîner une modification du comportement des produits de corrosion par rapport au fonctionnement à puissance stable. Des mesures sur réacteurs permettront d'en apprécier les conséquences et éventuellement d'adopter des dispositions permettant de les atténuer.

\section{b) Influence de circuits auxiliaires}

Les études théoriques effectuées à l'aide du code PACTOLE attribuent à certains circuits auxiliaires une importance non négligeable vis-à-vis des doses du personnel. Des mesures sont effectuées à la centrale de Fessenheim pour vérifier cette conclusion théorique, comprendre l'origine du phénomène et déterminer les actions correctives.

\section{c) Mise en arrêt à froid du réacteur}

La mise en arrêt à froid du réacteur est la cause de transferts importants d'activité de produits de corrosion qu'il convient de minimiser ou de favoriser selon leur origine de façon à réduire les débits de dose dans les zones d'intervention du personnel. 
Les nombreuses mesures effectuées depuis plusieurs années sur les réacteurs permettent et permettront encore de mieux comprendre les phénomènes physiques et d'améliorer les procédures d'exploitation.

\subsubsection{Prise en compte de l'expérience acquise au niveau des projets}

Les connaissances acquises concernant la nature et l'activité des dépôts actifs ont conduit à engager des études pour tenir compte de cette source à la conception et calculer de façon plus réaliste les débits de dose prévisibles.

\subsection{Dépôts de produits de fission}

Un programme expérimental (BOUFFON) est poursuivi depuis plusieurs années au CEA pour préciser les processus de sortie des produits de fission à l'extérieur des gaines au travers des ruptures et mesurer la contamination du fluide primaire en cherchant à reproduire différents modes de fonctionnement des réacteurs, en particulier le suivi de charge (voir paragraphe 2.1.5.). Par ailleurs, un important programme de mesures est effectué sur les centrales en service.

Comme pour les produits de corrosion, l'activité des produits de fission déposés semble prépondérante par rapport à celle contenue dans l'eau, pendant les périodes où le personnel intervient. Il était donc important de préciser l'incidence des dépôts de produits de fission sur les doses du personnel en cas de ruptures de gaines importantes.

Au cours du $2^{e}$ cycle de Bugey 2 , des dégradations importantes du combustible dues à un défaut des structures internes du réacteur ont conduit à atteindre la limite réglementaire de l'activité de l'eau primaire et à arrêter le réacteur. Une campagne de mesures a été effectuée à cette occasion. La participation des produits de fission aux doses du personnel a été évaluée à environ $6 \%$. Cette valeur faible, compte tenu du caractère exceptionnel de ce type d'incident, permet de considérer que les produits de corrosion sont en toutes circonstances, et de très loin, les principaux responsables des doses du personnel.

Les investigations seront, cependant, poursuivies aux cours des cycles de Bugey 2 pour examiner l'évolution de cette contamination, en particulier en ce qui concerne les transuraniens.

\subsection{Autres sources}

Les autres sources ont une incidence négligeable sur les doses du personnel, pour un fonctionnement normal du réacteur, pour des raisons diverses:

- activité faible ;

- forte activité mais bonnes protections ;

- temps de séjour négligeable du personnel à proximité.

Il est, cependant, nécessaire de bien les connaître pour s'assurer qu'elles ont effectivement peu de conséquences sur les doses. C'est le cas du tritium et plus généralement des produits de fission que l'on retrouve à la suite de transferts dans les circuits auxiliaires et éventuellement dans l'air des locaux. Les travaux d'un groupe de travail EdF-CEA ont permis de préciser, à partir de mesures effectuées dans les centrales par des équipes spécialisées 
des deux établissements, la nature et l'importance de ces transferts. Ces résultats ont, en outre, une importance particulière pour l'évaluation des conséquences d'accidents.

Le tritium a donné lieu à une étude particulière à la centrale de Tihange pour mieux préciser les sources de production et les voies de transfert. II est maintenant établi que ce radioélément n'occasionne pas de dose significative pour le personnel si on ne le laisse pas s'accumuler de façon trop importante dans le circuit primaire.

Les derniers développements des études concernant l'activité de l'eau en produits de fission et les limites réglementaires sur ce point ont conduit à prendre une attitude plus réaliste vis-à-vis des limites à adopter à la conception.

Les limites antérieures, trop élevées, conduisaient, dans certains cas, à des protections fixes surabondantes constituant une entrave aux déplacements rapides du personnel. II est important, dans ce domaine, que les protections fixes soient optimisées. La réduction des limites réglementaires pour l'activité de l'eau associée à la prise en compte de l'activité des dépôts, mentionnée précédemment, vont dans le sens de l'optimisation.

Parmi les mesures effectuées en centrale, celles relatives aux débits de dose dus aux neutrons ont permis de mieux connaître les sources dans les différents points du bâtiment réacteur et d'affiner les codes de calcul correspondants. Quelques campagnes particulières ont été effectuées pour déterminer, en un certain nombre de points, le spectre des neutrons.

L'étude des sources telle qu'elle vient d'être décrite précédemment, donne une meilleure compréhension des phénomènes physiques. Son principal objectif est la détermination des paramètres sur lesquels il serait possible d'agir pour en réduire l'activité.

\section{REDUCTION DE L'ACTIVITE DES SOURCES}

Le chapitre précédent explique pourquoi l'effort doit porter essentiellement sur la réduction de l'activité des dépôts. Pour ce faire, les moyens sont, en théorie, assez simples :

- réduction ou suppression des éléments chimiques susceptibles de s'activer sous rayonnement neutronique ;

- minimisation de l'activation pour des matériaux donnés ;

- élimination des dépôts actifs.

\subsection{Réduction ou suppression des éléments chimiques susceptibles de s'activer sous rayonnement neutronique : nature des matériaux}

Le principal élément indésirable est le cobalt 59 , stable, source de cobalt 60 par activation sous flux neutronique $\left({ }^{59} \mathrm{Co}(\mathrm{n}, \gamma){ }^{60} \mathrm{Co}\right)$ et, dans une moindre mesure, le cobalt 58 issu du nickel. Le cobalt est présent, en tant qu'impureté, dans la plupart des alliages en contact avec l'eau primaire.

Deux actions complémentaires sont envisageables : réduire la teneur en cobalt des matériaux et choisir parmi eux ceux dont le taux de relâchement 
est le plus faible. Les études effectuées pour sélectionner les zones du circuit primaire les plus sensibles ont conduit à mettre en évidence l'importance :

- de certaines structures des assemblages combustibles (grilles en inconel) (2) du fait de leur activité spécifique élevée et de leur solubilisation pendant les mises en arrêt à froid du réacteur ;

- du faisceau des tubes de générateur de vapeur du fait de leur surface importante ;

- des surfaces stellitées ${ }^{(3)}$ du fait de la très forte teneur en cobalt de ce matériau (50 à $60 \%$ ), en particulier celles du RCV ${ }^{(4)}$ où les conditions de fonctionnement sont défavorables.

\subsubsection{Réduction de la teneur en cobalt des matériaux}

- Stellites : des essais en laboratoire ont conduit à selectionner un type d'alliage de remplacement, le Colmonoy 5 : les essais se poursuivent actuellement sur certaines tranches nucléaires en vue de la qualification d'organes de sectionnement ainsi modifiés. Le gain escompté sur la dose collective annuelle est d'environ $5 \%$.

- Autres composants : une étude est en cours pour évaluer la possibilité et l'intérêt de réduire les fourchettes des spécifications admises pour certains composants particulièrement sensibles, tout en assouplissant celles des équipements en acier inoxydable qui ne participent pas à la formation de dépôts actifs.

\subsubsection{Réduction du taux de relâchement}

A paramètres physico-chimiques du milieu donnés, le taux de relâchement varie avec la nature du matériau. Un nouvel alliage (l'alliage 690) est envisagé pour remplacer l'alliage 600 utilisé actuellement pour les faisceaux de générateur de vapeur. Son taux de relâchement est sensiblement plus faible.

\subsection{Minimisation de l'activation pour des matériaux donnés}

Le but visé est de limiter :

- le relâchement du métal en contact avec l'eau primaire ;

- l'activation des produits de corrosion sous flux neutronique,

\subsubsection{Amélioration de la chimie du circuit primaire}

Les études expérimentales sur boucles ainsi que les calculs réalisés à l'aide du code PACTOLE ont abouti à l'élaboration d'une spécification de la teneur en lithine plus restrictive que celle du constructeur et conduisant à fonctionner tout au long du cycle (sauf à la fin), à un pH à chaud constant, fixé

(2) L'inconel est un alliage inoxydable largement utilisé dans les réacteurs PWR essentiellement pour les tubes de générateur de vapeur.

(3) Le stellite est un alliage utilisé pour sa grande dureté, en particulier pour résoudre des problèmes délicats de sièges de robinets et clapets.

(4) Circuit de contrôle volumétrique et chimique : il s'agit d'un circuit connecté directement au circuit primaire, assurant, entre autres, les fonctions de purification et de régulation du volume d'eau sous pression. 
de manière à minimiser les phénomènes de déposition sur les matériaux soumis au flux neutronique. Le gain escompté sur la dose collective annuelle est de 10 à $20 \%$.

Le fonctionnement à $\mathrm{pH}$ constant avec une faible tolérance conduit à une consommation de lithine importante : ce produit étant coûteux, des essais pourraient être entrepris pour juger de l'intérêt d'utiliser la potasse en remplacement de la lithine si la potasse ne se révèle pas, au cours d'essais préalables, plus agressive que la lithine vis-à-vis des matériaux en contact avec l'eau primaire. Bien qu'aucun effet bénéfique direct ne soit théoriquement attendu sur l'activité des circuits, ce point sera particulièrement suivi du fait que les deux seules centrales PWR en Europe (hors URSS) utilisant la potasse (LOVIISA en Finlande et TRINO en Italie) ont des doses assez faibles.

Par ailleurs, des études sont en cours, relatives à la possibilité de faciliter le réglage du $\mathrm{pH}$ en ajoutant le réactif (lithine ou potasse) dans les réservoirs d'eau d'appoint. Un contrôle plus précis du $\mathrm{pH}$ devrait à terme permettre de fonctionner à $\mathrm{pH}$ constant, même en fin de cycle, ce qui est théoriquement favorable à la réduction de l'activité des circuits.

\subsubsection{Grilles du combustible (5)}

Au fur et à mesure de la passation des nouveaux contrats de fourniture d'assemblages combustibles, les grilles en inconel seront remplacées par des grilles en zircaloy. Le relâchement de nickel activé sera nul. II devrait en résulter un gain de 10 à $15 \%$ sur la dose collective annuelle.

\subsubsection{Qualité de l'état de surface interne des équipements}

Les mesures de débit de dose sur les centrales en service ont récemment mis en évidence quelques anomalies dont on peut penser qu'elles ont pour origine l'état de surface interne des équipements correspondants. L'analyse de ce phénomène, qui semble avoir une influence aussi bien sur le relâchement que sur la redéposition des produits de corrosion, est en cours.

\subsection{Elimination des produits de corrosion}

Ils sont soit dans l'eau sous forme soluble ou insoluble, soit déposés sur les parois ; leur élimination passe par :

- la purification de l'eau primaire (par filtration, déminéralisation, etc.)

- la décontamination des parois internes des circuits.

\subsubsection{Purification de l'eau primaire}

- Les études et essais relatifs à une purification à chaud et à débit élevé au moyen d'un filtre électromagnétique ont été abandonnés du fait des incertitudes qui pèsent sur l'efficacité de cette technique, de son coût et des contraintes supplémentaires qu'elle entraînerait sur la conception et l'exploitation des tranches.

- Par contre, les études relatives à une purification à froid à débit élevé arrivent à leur terme. Cette purification permet d'éviter une redéposition des produits

(5) Les grilles sont des éléments permettant le maintien d'un ensemble de crayons et leur assurant une bonne tenue mécanique. 
de corrosion au cours des redémarrages faisant suite à un arrêt à froid de courte durée. Elle permet également, au cours des arrêts à froid, de réduire plus rapidement l'activité de l'eau primaire et, par conséquent, les débits de dose autour des circuits auxiliaires. Cette technique pourrait entraîner une réduction de 5 à $10 \%$ de la dose collective annuelle.

- Par ailleurs, il est envisagé pour les tranches du palier $1300 \mathrm{MW}$ de faire fonctionner, réacteur en service, la purification de l'eau primaire à son débit nominal tout au moins pendant les transitoires de puissance des réacteurs fonctionnant en base.

\subsubsection{Décontamination des parois internes des circuits actifs}

Elle peut faire appel à un processus mécanique ou chimique ; sá mise en œuvre peut être occasionnelle ou systématique.

\section{- Décontamination mécanique}

Elle n'intéresse que des portions limitées de circuits ou d'équipements, principalement :

- les parois des piscines : un outillage utilisant une lance à haute pression d'eau a été mis au point pour les tranches de $900 \mathrm{MW}$. II est en cours de perfectionnement ;

- les boîtes à eau des générateurs de vapeur : sur le même principe mais avec une addition de cristaux d'acide borique, le CEA termine la mise au point d'un outillage spécialisé ;

- le combustible : des essais et des études sont en cours au CEA pour juger de l'intérêt d'une décontamination par ultra-sons des assemblages de crayons de combustible au cours de l'arrêt annuel.

\section{- Décontamination chimique}

La décontamination chimique s'avère très délicate pour les réacteurs PWR, contrairement aux réacteurs BWR pour lesquels des applications fructueuses ont déjà été effectuées. Ceci s'explique du fait de la différence dans la nature chimique des dépôts due au caractère oxydant ou réducteur du milieu.

Les seuls procédés efficaces utilisent des solutions agressives pouvant entraîner ultérieurement des problèmes de corrosion dont on contrôle difficilement l'action. De ce fait, ils sont inutilisables, hormis pour des situations accidentelles exceptionnelles. Les procédés moins agressifs, dits de décontamination "douce", ont été expérimentés en boucle à La Hague (par le CEA) et au Canada (par London nuclear Ltd, LNL). Les résultats sont, jusqu'à présent, peu encourageants.

Les perspectives dans le domaine de la décontamination douce sont, pour le moment, limitées.

\section{- Procédures de mise en arrêt à froid des réacteurs}

Au cours de la mise en arrêt à froid des réacteurs PWR, les modifications des conditions physico-chimiques du fluide primaire entraînent des transferts importants de produits de corrosion. 
Des études et des essais sont engagés pour favoriser, au cours de cette phase d'exploitation, la mise en solution ou en suspension des dépôts et leur élimination par une purification rapide de l'eau primaire.

Les investigations portent, en particulier, sur la borication à chaud ${ }^{(6)}$ et le maintien à une température supérieure à $150^{\circ} \mathrm{C}$ du circuit primaire pendant une durée à déterminer.

La réduction de l'activité des sources ne représente qu'un aspect du problème de la réduction des doses : si leur activité est supposée immuable, il est possible d'agir sur le temps de séjour et le débit de dose sur le lieu de travail.

\section{REDUCTION DU DEBIT DE DOSE ET DU TEMPS DE SEJOUR}

Les actions engagées dans ce domaine sont extrêmement diversifiées. Elles portent sur de nombreux points tels que les protections biologiques, la préparation du travail, l'utilisation d'outillages spécialisés ou la formation du personnel.

\subsection{Protections biologiques}

EdF entreprend, pour chaque centrale, le dimensionnement des protections biologiques de façon que l'exploitation soit possible pour une activité de l'eau primaire égale à la limite réglementaire autorisée pour le fonctionnement du réacteur. Ces calculs permettent de s'assurer, en outre, que les circulations routinières sont situées dans des locaux à très faible débit de dose.

En particulier, une étude systématique des débits de dose liés aux opérations de déchargement, stockage et évacuation du combustible irradié a été effectuée. Cette étude a défini les épaisseurs minimales d'eau et de béton nécessaires en partie courante et dans tous les points singuliers du bâtiment réacteur pour limiter les doses délivrées lors de ces opérations.

Comme il a été dit précédemment, ces protections sont souvent une gêne au cours des opérations d'entretien. II est donc nécessaire d'optimiser leur usage en sachant que $75 \%$ des doses du personnel sont dues à l'entretien du matériel.

Ceci est particulièrement vrai dans le bâtiment réacteur où les protections neutroniques ne doivent pas créer une gêne trop importante pour les travaux d'entretien sur le circuit primaire. Des études de protections neutroniques adaptées sont en cours pour le palier de $300 \mathrm{MW}$. Elles ont pour but de limiter au maximum le flux de rayonnements sortant de la cuve, réacteur en service. Ce flux est, en effet, susceptible d'introduire un débit de dose non négligeable dans les zones accessibles, réacteur en service, ou de provoquer une activation des structures proches de la cuve qui pourra se traduire par des sources de rayonnements supplémentaires, réacteur à l'arrêt. Les moyens

(6) Le passage en arrêt à froid d'un réacteur PWR nécessite la mise en état " sous critique" du réacteur avec une certaine marge de sécurité ; celle-ci s'effectue par injection de bore (sous forme d'acide borique) dans le circuit primaire jusqu'à l'obtention d'une concentration relativement élevée (1500 à 2000 ppm); cette injection peut s'effectuer indifféremment " à chaud" ou au cours du refroidissement. 
de calcul utilisés pour ces études ont été validés à partir de mesures effectuées sur les centrales du palier de $900 \mathrm{MW}$ en service.

\subsection{Conception et installation du matériel fixe}

Des efforts importants sont effectués au niveau de l'installation du matériel afin de réduire les doses au personnel: la séparation des matériels actifs et inactifs, la séparation des matériels actifs entre eux, la commande à distance de certains équipements sont, par exemple, des moyens devenus routiniers. En complément, la réalisation d'une maquette des locaux et des équipements permet beaucoup plus facilement de mettre en évidence les problèmes qui peuvent se poser (circulations du personnel, accessibilité aux équipements, manutentions,etc.). Ainsi, un examen systématique des conditions d'intervention peut être fait dès le début des études.

Par ailleurs, un recueil de règles d'installation à l'usage des dessinateurs projeteurs a été rédigé. Ce document précise, entre autres, les règles à respecter pour l'installation du matériel afin d'assurer les meilleures conditions d'exploitation et d'entretien, en particulier vis-à-vis de la protection des personnes. Sa mise à jour périodique est réalisée pour tenir compte de l'expérience acquise dans les centrales en service.

\subsection{Utilisation de l'expérience des centrales en service}

Le démarrage des centrales du palier de $900 \mathrm{MW}$ permet de compléter l'expérience acquise sur des centrales plus anciennes comme Chooz et Tihange et de procéder à des modifications sur l'ensemble des tranches. Un système de gestion centralisée garantit qu'une modification, décidée sur une tranche et jugée de caractère générique, est bien réalisée sur toutes les autres tranches. Par ailleurs, une analyse systématique est effectuée, au plan national, pour les incidents jugés significatifs.

Les résultats des mesures de débit de dose et de dose effectuées par toutes les centrales dans des conditions identiques sont analysés pour suivre l'évolution de l'activité des circuits et la corréler aux doses du personnel. Cette analyse met en évidence les résultats favorables de telle ou telle centrale et constitue le point de départ de la recherche d'éléments conduisant à des réductions des doses.

Le fait d'avoir, dans la même société, à la fois les concepteurs et les exploitants permet de tirer le plus grand profit de l'expérience d'exploitation.

\subsection{Matériel mobile de radioprotection}

L'expérience acquise a permis de mieux définir les besoins et par suite de développer des matériels permettant à la fois une meilleure protection du personnel et des réductions de dose.

La mise en place du confinement des chantiers avec des moyens classiques conduirait à des doses élevées. Des équipements particuliers ont été mis au point, soit dans le domaine du confinement statique (tentes préfabriquées, panneaux gonflables,...), soit dans le domaine du confinement dynamique (ventilateurs et filtres associés sur chariot).

Par ailleurs, du matériel de mesure en continu de la contamination atmosphérique en aérosols dans des lieux où le débit d'équivalent de dose est de 
plusieurs dizaines de $\mu$ Sv. $h^{-1}$ permettra de limiter le port de protections respiratoires au strict nécessaire et par suite, d'éviter le supplément de dose occasionné par la gêne des opérateurs due à ces protections.

\subsection{Dosimètres individuels}

D'autre part, la mise au point de dosimètres électroniques individuels à affichage permanent et lecture directe, munis d'un indicateur lumineux de débit de dose, donne aux agents un moyen de suivre facilement l'évolution de leur dose et d'éviter de se tenir par inadvertance dans des zones à débit de dose élevé.

Enfin, la collecte automatique de ces doses et leur affectation au travail effectué est un moyen efficace pour :

- connaître chaque jour les doses individuelles et ainsi, mieux les répartir entre les agents d'une même spécialité ;

- connaître les doses prises sur les différentes opérations et, après analyse, déterminer les opérations sur lesquelles un effort de réduction des doses doit être engagé.

\subsection{Etude des interventions}

L'analyse des doses collectives fait apparaitre que les opérations d'entretien sont responsables d'environ $75 \%$ des doses annuelles subies par les travailleurs dans une centrale PWR. II est donc nécessaire que chacune de ces interventions soit soigneusement étudiée et préparée de façon à limiter le temps de séjour des opérateurs.

Pour ce faire, EdF a édité des guides destinés aux préparateurs, leur permettant de prendre en compte les problèmes de radioprotection dans la préparation du travail. Chaque intervention donne lieu à l'établissement d'une "gamme-type" dans laquelle sont décomposées les différentes opérations à effectuer. A chacune de ces opérations sont associés le temps nécessaire pour exécuter le travail, l'outillage, les moyens de manutention, les équipements de protection, le nombre et la qualification des agents, la procédure à utiliser, les précautions à prendre ainsi que la dose collective probable. A l'aide de ces gammes-types, le personnel a donc une idée précise du travail à effectuer, ce qui réduit le temps d'intervention.

Les interventions les plus coûteuses en dose donnent lieu à des études particulières afin de mettre au point un outillage spécialisé permettant soit le travail à distance, soit une réduction du temps d'intervention. C'est ainsi que la quasi-totalité des travaux systématiques effectués dans les boîtes à eau des générateurs de vapeur, où le débit de dose varie de 80 à $150 \mathrm{mSv} / \mathrm{h}$, sont d'ores-et-déjà mis en œuvre depuis l'extérieur de la boîte à eau.

Pour ce type d'opérations délicates, des maquettes à l'échelle 1 sont réalisées et utilisées, d'une part, pour la mise au point du matériel, d'autre part pour l'entraînement du personnel.

Des gammes d'intervention simplifiées sont élaborées dès la conception pour mettre en évidence les problèmes dus à l'entretien du matériel (manu- 
tentions, accessibilité, etc.). Elles permettent de prendre, avant la mise en service les mesures correctives nécessaires.

Par ailleurs, la multiplication des consignes et procédures de toutes sortes conduit le personnel à se démobiliser par rapport à ces prescriptions, ce qui peut entraîner des problèmes de sécurité. Un travail de réflexion est engagé, visant à la simplification des consignes et procédures.

\subsection{Formation du personnel}

La formation et la sensibilisation du personnel d'exécution et d'encadrement est un élément indispensable à la réduction des doses du personnel. Elle s'opère sur deux plans : la connaissance particulière du travail à effectuer et la connaissance des risques et des moyens de protection. Le premier point est obtenu à l'aide des gammes-types dont il a été question précédemment. Le deuxième point est acquis par le personnel au cours de conférences, travaux pratiques, stages et entraînement sur des chantiers-écoles [1].

\section{CONCLUSIONS}

Les chapitres précédents donnent un aperçu général de l'ampleur et de la diversité des actions entreprises. Cela est dû au fait que la radioprotection doit pénétrer tous les gestes à accomplir autour des équipements actifs. Elle doit devenir plus un état d'esprit qu'une science. C'est aussi la raison pour laquelle il est difficile de mesurer spécifiquement l'effet de telle ou telle action.

Certaines de ces actions ont un effet à court terme ; il est donc possible d'espérer dès à présent des améliorations. Le suivi attentif des centrales PWR en service permet d'améliorer les connaissances et de mettre au point l'organisation et les techniques. Mais il ne faut pas attendre, dans l'immédiat, de progrès spectaculaires : les progrès viendront d'un effort soutenu dans toutes les directions. A long terme, il est cependant possible d'espérer des gains plus substantiels de certaines méthodes de réduction de l'activité des dépôts.

\section{BIBLIOGRAPHIE}

[1] AYE L. Formation en radioprotection du personnel des centrales nucléaires d'Electricité de France. Radioprotection, 1980, 15, 183-192.

[2] JEANSON P. Actions entreprises pour la réduction des doses subies par les travailleurs des centrales PWR. Radioprotection, 1978, 13, 167-175. 